\title{
PROJECTIVE DESCRIPTION OF SOME PLANE SEXTIC CURVES DERIVED FROM CONICS AS BASE CURVES
}

\author{
SR. INGONDA MARIA VON MEZYNSKI, S.SP.S.
}

Introduction. Comparatively few of the plane sextic curves are known. None of the papers written about them gives a complete treatment or a classification of these curves. The sextics appear more or less isolated in the different treatises. Apparently the methods employed produce only a very limited number of sextics.

The projective method of deriving higher plane curves described by Dr. H. P. Pettit in his Projective description of some higher plane curves $^{1}$ allows a more systematical study of sextics and especially a direct construction point by point.

It is the purpose of this paper to give a projective description of some of the sextics that can be derived by this method, if conics are the base curves. The number of types of these curves which could be generated by this method is here limited to 354 by giving the triangle of reference special positions with respect to the base conics.

Discussion of the general curve derived from conics as base curves. The method described by Dr. Pettit in his Projective description of some higher plane curves is as follows:

Let there be given two curves, $C_{1}$ of order $n$ and $C_{2}$ of order $m$, two projective pencils $A_{1}, A_{3}$ and one connecting pencil $A_{2}$ in the plane. Any line on $A_{1}$ cuts $C_{1}$ in $n$ points and the $n$ lines joining these points to $A_{2}$ cut $C_{2}$ in $m n$ points, which determine $m n$ lines on $A_{3}$ cutting the line on $A_{1}$ in points of the generated curve.

For the present purpose let $A_{1}, A_{2}, A_{3}$ be the vertices of the triangle of reference and let the equation of the pencil on $A_{1}$ be

$$
x_{2}-\lambda x_{3}=0,
$$

that of the pencil on $A_{2}$ be

$$
x_{1}-\mu x_{3}=0,
$$

and that of the pencil on $A_{3}$ be

$$
x_{1}-\nu x_{2}=0 \text {. }
$$

Let the equations of the base conics be

Presented to the Society, August 14, 1944; received by the editors July 11, 1944.

${ }^{1}$ Dr. H. P. Pettit, Projective description of some higher plane curves, Tôhoku Math. J. vol. 28 (1927-1928) pp. 72-79. 


$$
\begin{aligned}
u_{0} x_{1}^{2}+u_{1} x_{1}+u_{2} & =0, \\
v_{2}+v_{1} x_{3}+v_{0} x_{3}^{2} & =0,
\end{aligned}
$$

where

$$
\begin{array}{ll}
u_{0}=a, & v_{0}=c^{\prime}, \\
u_{1}=2\left(g x_{3}+h x_{2}\right), & v_{1}=2\left(g^{\prime} x_{1}+f^{\prime} x_{2}\right), \\
u_{2}=b x_{2}^{2}+c x_{3}^{2}+2 f x_{2} x_{3}, & v_{2}=a^{\prime} x_{1}^{2}+b^{\prime} x_{2}^{2}+2 h^{\prime} x_{1} x_{2} .
\end{array}
$$

If we eliminate $x_{1}, x_{2}, x_{3}$ from (1), (2) and (4), (4) takes the form $f(\mu, \lambda, 1)=0$, and if we eliminate $x_{1}, x_{2}, x_{3}$ from (2), (3) and (5), (5) takes the form $\phi(\mu \nu, \mu, \nu)=0$. Then eliminating $\mu$ from $f(\mu, \lambda, 1)=0$ and $\phi(\mu \nu, \mu, \nu)=0$ and expressing $\lambda$ and $\nu$ again in terms of $x_{1}, x_{2}, x_{3}$ from (1) and (3) we obtain the equation of the generated curve

(6) $\left(u_{0} v_{0} x_{1}^{2} x_{3}^{2}-u_{2} v_{2}\right)^{2}-x_{1} x_{3}\left(u_{0} v_{1} x_{1} x_{3}-u_{1} v_{2}\right)\left(u_{1} v_{0} x_{1} x_{3}-u_{2} v_{1}\right)=0$.

Hence the general curve derived by this method, if conics are the base curves, is an octic curve. It follows from the equation (6) that the octic passes through the intersections of the quartic

$Q$ :

$$
u_{0} v_{0} x_{1}^{2} x_{3}^{2}-u_{2} v_{2}=0
$$

with the cubic

$C_{1}$ :

$$
u_{0} v_{1} x_{1} x_{3}-u_{1} v_{2}=0
$$

and the cubic

$C_{2}$ :

$$
u_{1} v_{0} x_{1} x_{3}-u_{2} v_{1}=0 .
$$

The quartic $Q$ counted twice is generated by the same method, if $A_{1}$ is the pole of $x_{1}=0$ with respect to the first base conic (4) and $A_{3}$ is the pole of $x_{3}=0$ with respect to the second base conic (5). The base curves for the generation of the quartic are then

$$
\begin{gathered}
u_{0} x_{1}^{2}+u_{2}=0, \\
v_{2}+v_{0} x_{3}^{2}=0 .
\end{gathered}
$$

The conic (a) passes through the intersections of $x_{1}=0, u_{1}=0$ with the conic (4) and it is tangent to the lines $u_{2}=0$ at the intersections of $x_{1}=0$ with (4). The conic (b) passes through the intersections of $x_{3}=0, v_{1}=0$ with (5) and it is tangent to the lines $v_{2}=0$ at the intersections of $x_{3}=0$ with the conic (5).

By the same method the cubic $C_{1}$ is generated, if 


$$
\begin{gathered}
u_{0} x_{1}+u_{1}=0 \\
v_{2}+v_{1} x_{3}=0
\end{gathered}
$$

are the base curves. The line (a) passes through the intersection of $x_{1}=0$ with $u_{1}=0$. It cuts (4) in the points in which $u_{2}=0$ cuts (4) but not in the points where $x_{1}=0$ cuts (4) at the same time. The conic (b) has contact with (5) at both the intersections of $x_{3}=0$ with (5). It passes through $A_{3}$.

The cubic $C_{2}$ is generated, if

$$
\begin{aligned}
u_{1} x_{1}+u_{2} & =0, \\
v_{1}+v_{0} x_{3} & =0
\end{aligned}
$$

are the base curves. The conic (a) passes through $A_{1}$ and has contact with (4) at both the intersections of $x_{1}=0$ with (4). The line (b) passes through the intersection of $x_{3}=0$ with $v_{1}=0$. It cuts (5) where $v_{2}=0$ cuts (5) but not at the point where $x_{3}=0$ cuts (5).

Thus the quartic and the cubics can be constructed easily and the intersections of these curves can be found geometrically. Algebraically the intersections of the quartic with the cubic $C_{1}$ are given by

$$
\text { (7) } x_{2}^{2} v_{2}\left\{\left[b\left(2 g v_{2}-a x_{1} v_{1}\right)-2 f h v_{2}\right]^{2}-4 h^{2} v_{2}\left[\left(f^{2}-b c\right) v_{2}+a b c^{\prime} x_{1}^{2}\right]\right\}=0 \text {. }
$$

Since a quartic and a cubic intersect in twelve points, four of the intersections are at $A_{3}$. Two intersections are at $A_{1}$, two given by $v_{2}=0$ are where $x_{3}=0$ cuts the conic (5) and the other four given by the expression in the braces are outside of the triangle of reference.

The intersections of $Q$ with $C_{2}$ are given by

$$
\left(7^{\prime}\right) x_{1}^{2} x_{2}^{4}\left\{\left[b\left(2 g v_{2}-a x_{1} v_{1}\right)-2 f h v_{2}\right]^{2}-4 h^{2} v_{2}\left[\left(f^{2}-b c\right) v_{2}+a b c^{\prime} x_{1}^{2}\right]\right\}=0 \text {. }
$$

Hence two of them are at $A_{3}$, four at $A_{1}$, two where $x_{1}=0$ cuts the conic (4) and four given by

(8) $\left[b\left(2 g v_{2}-a x_{1} v_{1}\right)-2 f h v_{2}\right]^{2}-4 h^{2} v_{2}\left[\left(f^{2}-b c\right) v_{2}+a b c^{\prime} x_{1}^{2}\right]=0$

are outside of the triangle of reference. Comparing (7) with $\left(7^{\prime}\right)$, we see that the quartic cuts the cubics in the same points outside of the triangle of reference.

The intersections at $A_{1}$ and $A_{3}$ are quadruple points of the octic, for $x_{2}=0$ cuts (6) in $x_{1}^{4} x_{3}^{4}=0$. The line $x_{1}=0$ cuts (6) in the double points given by $u_{2}^{2}=0$, and $x_{3}=0$ cuts the octic in the double points given by $v_{2}^{2}=0$. These four double points are where $x_{1}=0$ cuts the conic (4) and where $x_{3}=0$ cuts the conic (5). The four intersections 
outside of the triangle of reference are also double points of the octic, for any line satisfying (8) cuts the octic in points given by

$$
\left(k_{1} x_{1}+k_{2} x_{3}\right)^{2}\left(K_{1}^{2}-x_{1} x_{3} K_{2} K_{3}\right)=0,
$$

where $K_{1}$ is an expression of the third degree in $x_{1}, x_{3}$ and $K_{2}, K_{3}$ are of the second degree in $x_{1}, x_{3} . k_{1} x_{1}+k_{2} x_{3}=0$ gives a point of intersection of a line given by (8) with the octic or with the quartic and the cubics. But this point is a double point of the octic as follows from the above equation. Thus we have the following theorem.

THEOREM I. If the base curves are conics, the curve generated by this method is generally an octic curve with two quadruple points and eight double points.

This octic curve will degenerate into sextic curves, if (a) $A_{1}$ is on the first base conic (4) or what is the same if $A_{3}$ is on the second base conic (5); (b) if $A_{2}$ is on one of the base conics; (c) if the line $A_{1} A_{3}$ passes through two intersections of the base conics. ${ }^{2}$ These degenerations of the octic produce only sextics with deficiencies zero and one. The singularities occurring are quadruple points and double points. If by this method sextics with a higher deficiency can be generated when conics are the base curves has not yet been determined. Likewise it remains a question whether or not there exists the possibility of obtaining by this method sextics with triple points when conics are the base curves.

Sextics with one quadruple point and three double points. Let $A^{1}$ be on the first base conic (4). Then the equation (6) becomes:

$$
v_{2}\left[u_{2}^{2} v_{2}+u_{1} x_{1} x_{3}\left(u_{1} v_{0} x_{1} x_{3}-u_{2} v_{1}\right)\right]=0 .
$$

Hence the lines $v_{2}=0$ break off from the octic and the generated curve is a sextic. Since the lines $v_{2}=0$ split off from the octic, $A_{3}$ becomes a double point and the intersections of $x_{3}=0$ with (5) become ordinary points of the curve. The equation (8) reduces to $v_{2}^{2}=0$. Therefore there are no double points outside of the triangle of reference.

THEOREM II. The generated curve is a plane sextic with one quadruple point and three double points, if $A_{1}$ is on the first base conic. The equation of this sextic is

$$
u_{2}^{2} v_{2}+u_{1} x_{1} x_{3}\left(u_{1} v_{0} x_{1} x_{3}-u_{2} v_{1}\right)=0 .
$$

Just as in the construction of conics by means of projective pencils

2 Ibid. pp. 77-78. 
the line corresponding to the line joining one vertex to the other is a tangent to the conic at the other vertex, so also here. The tangents to the sextic at the quadruple point $A_{1}$ are the lines corresponding to $A_{3} A_{1}$ and the tangents at the double point $A_{3}$ are the lines corresponding to $A_{1} A_{3}$ in the projectivity set up. The equation of the tangents to the sextic at $A_{1}$ is

$$
c^{\prime} u_{1}^{2} x_{3}^{2}-2 g^{\prime} u_{1} u_{2} x_{3}+a^{\prime} u_{2}^{2}=0 .
$$

Let the line $A_{3} A_{1}$ cut the second base conic in $B_{1}$ and $B_{2}$. Then there can be distinguished seven kinds of quadruple points as follows:

1. Quadruple point of the 1st kind: All four tangents are real and distinct. The lines $A_{2} B_{1}$ and $A_{2} B_{2}$ cut the first base conic in real and distinct points.

2. Quadruple point of the 2nd kind: Two of the tangents coincide and the others are real and distinct. One of the lines $A_{2} B_{1}$ or $A_{2} B_{2}$ is tangent to the first base conic and the other one cuts it in real and distinct points.

3. Quadruple point of the 3rd kind: Two pairs of tangents coincide. The two lines $A_{2} B_{1}$ and $A_{2} B_{2}$ are tangent to the first base conic.

4. Quadruple point of the 4th kind: All four tangents coincide. The lines $A_{2} B_{1}$ and $A_{2} B_{2}$ coincide and are tangent to the first base conic.

5. Quadruple point of the 5th kind: Two of the tangents coincide and the other two are imaginary. One of the lines $A_{2} B_{1}$ or $A_{2} B_{2}$ is tangent to the first base conic and the other one cuts it in imaginary points.

6. Quadruple point of the 6th kind: Two of the tangents are real and distinct and the other two are imaginary. One of the lines $A_{2} B_{1}$ or $A_{2} B_{2}$ cuts the first base conic in real and distinct points and the other one cuts it in imaginary points.

7. Quadruple point of the 7th kind: All four tangents are imaginary. $A_{2} B_{1}$ and $A_{2} B_{2}$ cut the first base conic in imaginary points.

Let $A_{1} A_{3}$ cut the first base conic in $B_{3}$ and let $A_{2} B_{3}$ cut the second base conic in $B_{4}$ and $B_{5}$. Then $A_{3} B_{4}$ and $A_{3} B_{5}$ are the tangents to the sextic at $A_{3}$. Their equation is

$$
4 g^{2} v_{0} x_{1}^{2}-2 c g v_{1} x_{1}+c^{2} v_{2}=0 .
$$

The tangents are real and distinct, coincident, or imaginary or $A_{3}$ is a node, cusp, or conjugate point according as

$$
c^{2}\left(h^{\prime 2}-a^{\prime} b^{\prime}\right)+4 g^{2}\left(f^{\prime 2}-b^{\prime} c^{\prime}\right)-4 c g\left(f^{\prime} h^{\prime}-b^{\prime} g^{\prime}\right)>0,=0,<0,
$$

or as $A_{2} B_{3}$ cuts the second base conic in real and distinct, coincident, or imaginary points. 
The equations of the tangents to the sextic at the double points $P_{1}, P_{2}$, the intersections of $x_{1}=0$ with the first base conic, show that $P_{1}$ and $P_{2}$ are nodes, cusps, or conjugate points according as

$$
f^{\prime 2}-b^{\prime} c^{\prime}>0,=0,<0,
$$

or as $x_{1}=0$ cuts the second base conic in real and distinct, coincident, or imaginary points. $P_{1}$ and $P_{2}$ are consecutive, if $x_{1}=0$ is tangent to the first base conic. It follows from the expansion and the equation of the tangents at this point that this consecutive double point is a tacnode, node-cusp, or a tacnode isolated according as $x_{1}=0$ cuts the second base conic in real and distinct, coincident, or in imaginary points.

Likewise $A_{3}$ is a tacnode, node-cusp, or a tacnode isolated, if $A_{3}$ is on the first base conic and $x_{1}=0$ cuts the second base conic in real and distinct, coincident, or in imaginary points.

All three double points coincide at $A_{3}$, if $A_{3}$ is on the first base conic and $x_{1}=0$ is tangent to this conic at $A_{3} . A_{3}$ is then an oscnode, tacnode-cusp, or an oscnode isolated according as $x_{1}=0$ cuts the second conic in real and distinct, coincident, or in imaginary points.

$P_{1}$ and $P_{2}$ are imaginary, if $x_{1}=0$ cuts the first base conic in imaginary points.

The combinations of all these conditions for the different kinds of multiple points result in the following different types of sextics:

63 sextics with one quadruple point and three real and distinct double points;

57 sextics with one quadruple point, two consecutive double points and one real and distinct double point;

18 sextics with one quadruple point and three consecutive double points;

21 sextics with one quadruple point, one real and two imaginary double points.

Sextics with one quadruple point and four double points. Let $A_{1}$ be again on the first base conic (4) and in addition let $x_{1}-x_{3}=0$ on $A_{2}$ be a common tangent of the base conics. Then

$$
b=(f+h)^{2} /(c+2 g), \quad b^{\prime}=\left(f^{\prime}+h^{\prime}\right)^{2} /\left(a^{\prime}+c^{\prime}+2 g^{\prime}\right),
$$

and two ordinary points coincide forming a double point $P_{3} .^{3}$ Thus we have the following theorem.

THEOREM III. The generated curve is a plane sextic with one quad-

Ibid. p. 78. 
ruple point and four double points, if $A_{1}$ is on the first base conic and $A_{2}$ is on a common tangent of the base conics.

The only double point to be investigated here is $P_{3}$. The equation of the tangents at this point and the construction show that $P_{3}$ is a node or a conjugate point according as $A_{2}$ is between the points of contact of the common tangent with the conics or outside of this segment.

If we give the triangle of reference all possible positions it may take, there result the following different types of sextics:

63 types with one quadruple point and four real and distinct double points;

41 types with one quadruple point, two consecutive and two real and distinct double points;

12 types with one quadruple point, three consecutive double points and one real and distinct double point;

27 types with one quadruple point, two real and distinct double points and two imaginary double points.

The sextic generated when $A_{2}$ is on one base conic is essentially the same as the sextic discussed in this section.

Sextics with nine double points. Let $A_{1} A_{3}$ pass through two intersections of the base conics and let the polar of $A_{1}$ with respect to the first base conic and the polar of $A_{3}$ with respect to the second base conic pass through $A_{2}$. Then $a^{\prime}=a, c^{\prime}=c, g^{\prime}=g, f^{\prime}=h=0$. The double line $x_{2}^{2}=0$ breaks off from the octic, for it is a factor of the equation (6) if the above values are substituted in (6). The generated curve is therefore a sextic. It has double points at $A_{1}$ and $A_{3}$, at the intersections $P_{1}, P_{2}$ of $x_{1}=0$ with the first base conic (4) and at the intersections $P_{3}, P_{4}$ of $x_{3}=0$ with the second base conic (5). The equation (8) reduces to

$$
x_{2}\left(b^{\prime} x_{2}+2 h^{\prime} x_{1}\right)^{2}=0 .
$$

Hence the curve has three double points outside of the triangle of reference, one of which is consecutive with the double point $A_{1}$. Two of them are on the line $b^{\prime} x_{2}+2 h^{\prime} x_{1}=0$. This line cuts the sextic in the points given by

$$
x_{2}^{4}\left(b x_{2}+2 f x_{3}\right)^{2}=0
$$

that is, in two consecutive double points at $A_{3}$ and in one double point $P_{5}$ on the line $b x_{2}+2 f x_{3}=0$.

THEOREM IV. The generated curve is a plane sextic with two pairs of two consecutive and five real and distinct double points, if $A_{1} A_{3}$ passes 
through two intersections of the base conics and if the polar lines of $A_{1}$ with respect to the first base conic and of $A_{3}$ with respect to the second base conic pass through $A_{2}$.

The equations of the tangents at $A_{1}$ and $A_{3}$ and the expansion at these points show that $A_{1}$ and $A_{3}$ are tacnodes or tacnodes isolated, according as the line $A_{1} A_{3}$ passes through two real and distinct or imaginary intersections of the base conics. Since the polar lines of $A_{1}$ with respect to the first base conic and of $A_{3}$ with respect to the second base conic pass through $A_{2}, A_{1} A_{3}$ may not pass through a point of contact of the base conics. For this would cause $A_{2}$ to be on the conics and consequently a degeneration of the sextic would follow. Hence $A_{1}$ and $A_{3}$ cannot be node-cusps.

The tangents to the sextic at $P_{1}$ and $P_{2}$ are real and distinct, coincident, or imaginary, that is, $P_{1}$ and $P_{2}$ are nodes, cusps, or conjugate points according as $-b^{\prime} c>0,=0,<0$. But $b^{\prime}$ and $c$ may not be zero. Therefore $P_{1}$ and $P_{2}$ are nodes or conjugate points according as $x_{1}=0$ cuts the second base conic in real and distinct or in imaginary points.

Similarly $P_{3}$ and $P_{4}$ are nodes or conjugate points according as $-a b>0,=0,<0$, or as $x_{3}=0$ cuts the first base conic in real and distinct or in imaginary points.

$P_{1}$ and $P_{2}$ are consecutive and form a tacnode or a tacnode isolated, if $x_{1}=0$ is tangent to the first and cuts the second base conic in real and distinct or in imaginary points. Likewise $P_{3}$ and $P_{4}$ coincide forming a tacnode or a tacnode isolated, if $x_{3}=0$ is tangent to the second and cuts the first base conic in real and distinct or in imaginary points. $P_{1}$ and $P_{2}, P_{3}$ and $P_{4}$ are imaginary double points, if $x_{1}=0$ cuts the first and $x_{3}=0$ cuts the second base conic in imaginary points.

The double point $P_{5}$ has the coordinates $b^{\prime} f,-2 f h^{\prime}, b h^{\prime}$. It follows from the equation of the tangents to the sextic at $P_{5}$ that $P_{5}$ is a node or a conjugate point according as $x_{2}=0$ passes through two real intersections of the base conics or through two imaginary ones. $P_{5}$ coincides with $A_{1}$, if $h^{\prime}=0$, that is, if the polar of $A_{1}$ with respect to the second base conic passes through $A_{2} . A_{1}$ is then an oscnode or an oscnode isolated according as $x_{2}=0$ passes through two real or through two imaginary intersections of the base conics.

Taking into consideration all the possible combinations of the different kinds of double points discussed, there can be generated the following types of sextics:

6 types with two pairs of two consecutive and five real and distinct double points;

6 types with three pairs of two consecutive and three real and distinct double points; 
2 types with four pairs of two consecutive and one real and distinct double point;

4 types with three consecutive, two consecutive and four real and distinct double points;

2 types with three consecutive, two pairs of two consecutive and two real and distinct double points;

3 types with two imaginary, two pairs of two consecutive and three real and distinct double points;

2 types with two imaginary, three pairs of two consecutive double points and one real and distinct double point;

3 types with two imaginary, three consecutive, two consecutive and two real and distinct double points;

1 type with two imaginary, three consecutive and two pairs of two consecutive double points;

2 types with four imaginary, two pairs of two consecutive double points and one real and distinct double point;

1 type with four imaginary, three consecutive and two consecutive double points.

Sextics with ten double points. Let the position of the triangle of reference with regard to the base conics be chosen as in the preceding section and let in addition $A_{2}$ be on a common tangent of the conics. The generated sextic will have one more double point $P_{6}$ caused by the coincidence of two ordinary points.

THEOREM V. The generated curve is a plane sextic having two pairs of two consecutive and six real and distinct double points, if $A_{1} A_{3}$ passes through two intersections of the base conics, if the polar lines of $A_{1}$ with respect to the first and of $A_{3}$ with respect to the second base conic pass through $A_{2}$, and if $A_{2}$ is on a common tangent of the base conics.

The double point $P_{6}$ has the coordinates $h^{\prime},-(a+c+2 g), f$, if $x_{1}-x_{3}=0$ is the common tangent. It follows from the equation of the tangents to the sextic at this point that $P_{6}$ is a node or a conjugate point according as $A_{2}$ is between the points of contact of the common tangent with the conics or outside of this segment.

The conditions for the different kinds of double points of the other singularities of the curve are the same as those discussed in the last section. Thus if we give the triangle of reference all possible positions with regard to the base conics, there result the following types of sextics:

7 types with two pairs of two consecutive and six real and distinct double points; 
2 types with three pairs of two consecutive and four real and distinct double points;

1 type with four pairs of two consecutive and two real and distinct double points;

4 types with two imaginary, two pairs of two consecutive and four real and distinct double points;

3 types with two imaginary, three pairs of two consecutive and two real and distinct double points;

3 types with four imaginary, two pairs of two consecutive and two real and distinct double points.

A far greater number of sextics having nine or ten double points can be derived by this method, if the polar lines of $A_{1}$ with respect to the first and of $A_{3}$ with respect to the second base conic do not pass through $A_{2}$. But then it is very difficult to find the geometrical conditions for the different kinds of double points, which is important for the construction of these curves.

Holy Ghost Academy 